\title{
Application of mechanical heat treatment for the recovery of plastics as
} energy resource

\author{
Jyi-Yeong Tseng ${ }^{1}$, Chia-Chi Chang ${ }^{1}$, Zang-Sie Hung ${ }^{1}$, Yen-Chi Wang ${ }^{1}$, Dar-Ren $\mathrm{Ji}^{1}$, \\ Chun-Han $\mathrm{Ko}^{2}$, Yi-Hung Chen ${ }^{3}$, Je-Lueng Shie ${ }^{4}$, Yuan-Shen $\mathrm{Li}^{4}$, Chungfang Ho Chang ${ }^{5}$, \\ Sheng-Wei Chiang ${ }^{1}$, Shi Guan Wang ${ }^{6}$, Kuang Wei Liu ${ }^{6}$, Ching-Yuan Chang, ${ }^{1, *}$ \\ ${ }^{1}$ Graduate Institute of Environmental Engineering, National Taiwan University, Taipei, Taiwan \\ ${ }^{2}$ School of Forestry and Resource Conservation, National Taiwan University, Taipei, Taiwan \\ ${ }^{3}$ Department of Chemical Engineering and Biotechnology, National Taipei University of Technology, Taipei, \\ Taiwan \\ ${ }^{4}$ Department of Environmental Engineering, National I-Lan University, I-Lani, Taiwan \\ ${ }^{5}$ Department of International Trade, Chung Yuan Christian University, Chung-Li, Taiwan \\ ${ }^{6}$ Environmental Analysis Laboratory, Environmental Protection Administration, Chung-Li, Taiwan \\ * Corresponding author. Tel: +886 223638994, Fax: +886 223638994, E-mail: cychang3@ntu.edu.tw
}

\begin{abstract}
The mechanical heat treatment (MHT) is one of the pre-treatment alternatives for conditioning the municipal solid waste (MSW) before its further separation, recovery and reuse. The MHT would result in the change of properties of constituents of MSW, making it suitable for separation. For example, the plastics may be softened and shrunken. Therefore, the MSW via the pre-MHT can be more easily separated into various fractions of resources such as metals, plastics, compost-like and primary refuse derived fuel (RDF) or bio-char for further re-utilization.

The objective of this study was to examine the efficiency and effective of energy recovery and volume downsize of plastics via MHT process. The commonly used plastic, high-density polyethylene (HDPE) was tested. The changes of weight, triple components, true density and calorific value of target plastic before and after the MHT with saturated steam at 100,150 and $180{ }^{\circ} \mathrm{C}$ were examined. The effects of temperature on the performance of MHT were assessed. The results indicated that an increase of MHT temperature induces more significant shrinkage and higher volume density, enhancing its feasibility for the separation from non-plastic materials. The information obtained in this study is useful for the rational design and proper operation of MHT system for treating the used plastics in the MSW and separating it for the re-utilization as energy resource.
\end{abstract}

Keywords: Energy resource, Mechanical heat treatment, MHT, Municipal solid waste, MSW.

\section{Introduction}

The ultimate goal of the waste management aims at the complete collection of the garbage, proper treatment for the thorough energy recovery and material reuse targeting at a sustainable utilization of all resources. Traditional waste treatments processes include landfill, incineration and recycling. Incineration is a process which directly combust or incinerate the waste to generate heat and then recycle the energy through the production of steam or hot water with the help of heat recovering facilities. The incineration residues including sand, ceramic, glass, metal and little incomplete combustion of organic matter are sent to landfill. The process of incineration not only wastes a significant amount of reusable energy, but also produces secondary pollutants such as ash dregs or fly ash [1-3]. Further, if the plastics are directly recycled without any pretreatment, it will cause the lack of storage space and the consumption of transportation expense. Therefore, the new generation of energy recovery technique of the waste to replace incineration must take into consideration all kinds of factors such as the complex varieties and properties of the waste, the availability of waste as a resource, the technique for its transportation and storage, the constrain of economics and relevant regulations and the energy or environmental benefit for the whole systems. Thereafter, in addition to the enhancement of performance and the reduction of the cost, it has 
become a trend converting the waste into energy of different forms. According to a report of Environmental Protection Administration of Taiwan (TEPA) in 2008 for the Municipal solid waste (MSW) composition, there are $44.54 \%$ of paper, $2.63 \%$ of fibrous cloth, $1.99 \%$ of wood, bamboo and fallen leaves, $30.56 \%$ of kitchen waste, $17.28 \%$ of plastics, $0.36 \%$ of leather and rubber, $0.33 \%$ of ferric metals, $0.33 \%$ of non-ferric metals, $1.11 \%$ of glass, $0.5 \%$ of ceramic sands and $0.48 \%$ of other combustible materials [4]. These data reveals that there is a high content of plastics in the MSW. With certain kinds of pretreatment techniques, the separation of MSW and the followed resources recovery and reutilization may become more complete and efficient. The so called cooking assorting or mechanical heat treatment (MHT) is one of the techniques that are worthwhile to be evaluated [5,6]. The MHT would result in the change of properties of constituents of MSW, making it suitable for separation. For example, the mass of non-plastics decreases while the volume density increases. The cellulose and hemi-cellulose of biomass in MSW are decomposed for easy torrefaction. The plastics may be softened and shrunken. Thus, the objective of this study was to examine the efficiency and effective of energy recovery and volume downsize of plastics via MHT process. In this research, we investigated over the changes of weight, triple components, true density and calorific value of high density polyethylene (HDPE) before and after the cooking process for the reference need in plastic recycling from MSW via cooking separation process.

\section{Methodology}

\subsection{Devices}

Cooking apparatus used for this experiment is illustrated in Fig. 1. This apparatus includes a steam generator that can produce saturated steam and a high temperature (398-593 K) and high pressure (5-205 $\mathrm{kg} \mathrm{cm}^{-2}$ ) autoclave equipped with stirring accessory. The autoclave is made by stainless steel 316 with inner volume of $1.5 \mathrm{~L}$. The stirring axes are composed by outer and inner axes. The inner axis is enclosed in the outer axis and driven by the outer axis with magnetic force. The bearing is cooled by cooling water. The outer axis is connected to $0.25 \mathrm{hp}$ motor. The upper cap has six holes with three for thermal couple, pressure gauge and release valve, while three for spare port. The steam generator is constructed by an autoclave and an oven. The heating wire of oven is made of nichrome. The oven temperature is controlled by a proportional-integral-differential (PID) controller with K-type thermal couple in the vessel.

\subsection{Experimental conditions}

HDPE, the most common plastic that can be found in MSW for the experiment, was selected. Then the cooking experiments was performed 60 minutes with $100,150\left(\mathrm{P}_{\mathrm{g}}=5.15-5.4 \mathrm{~kg} \mathrm{~cm}^{-}\right.$ $\left.{ }^{2}\right)$ and $180{ }^{\circ} \mathrm{C}\left(\mathrm{Pg}=8.05-10.25 \mathrm{~kg} \mathrm{~cm}^{-2}\right)$ saturated high temperature steams via cooking apparatus. The dry weight changes, triple components (including water, ash and combustible components), true density and calorific value for the HDPE before and after the cooking process were examined.

\subsection{Analytical methods}

The thermal stability of HDPE was analyzed by the thermal gravity analysis (TGA) (model TGA51, Shimadzu Co., Kyoto, Japan). The calorific value was measured by a calorimeter (model 6775, Parr Instrument Company, Moline, Illinois, USA). The triple components were analyzed by a high-temperature oven (model muffle furnace DF 40, Deng Yng Co., Taipei, Taiwan). 


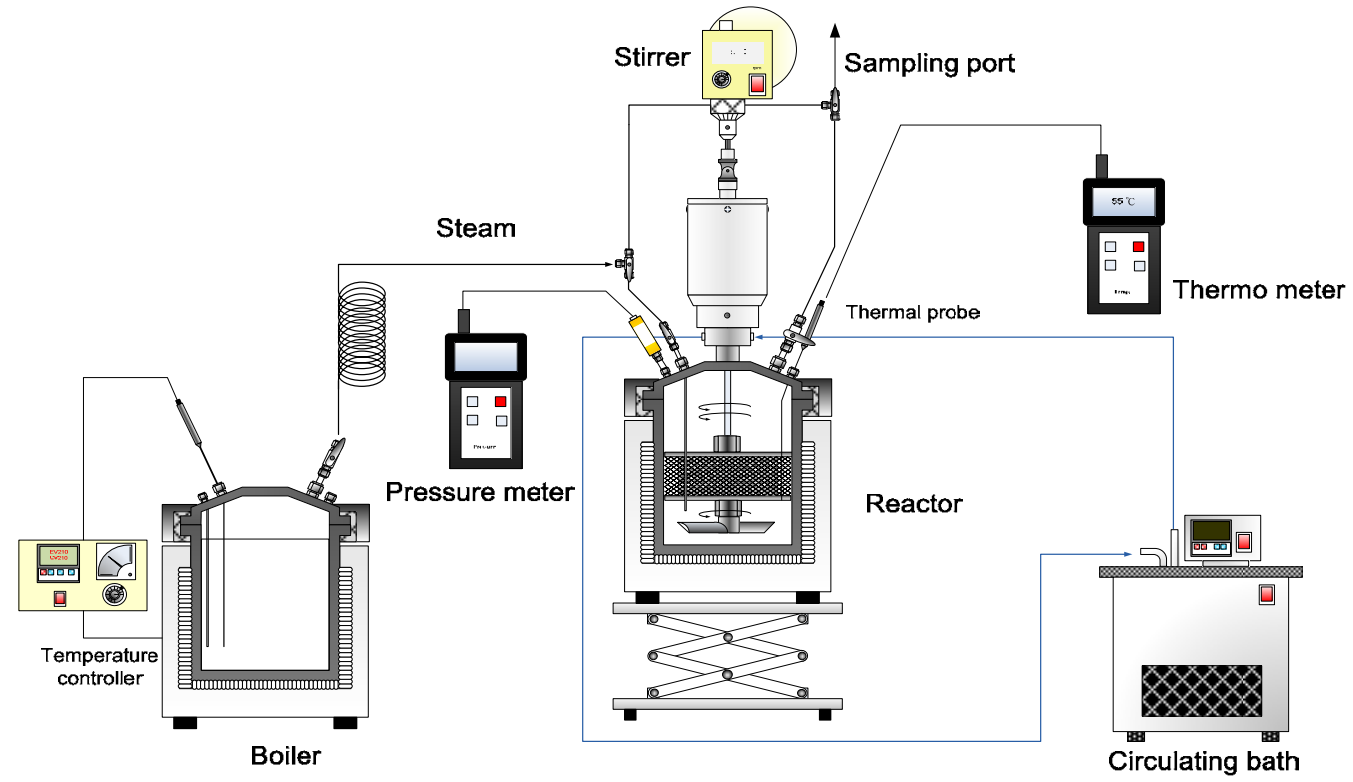

Fig. 1. Mechanical heat treatment (MHT) apparatus used for the recovery of plastics as energy resource.

\section{Results}

\subsection{TGA of HDPE}

TGA has proven to be a useful and efficient technique of the estimation of thermal stabilities of plastics. TGA is also widely used in studying pyrolytic processes. In this research, we investigated over the TGA to observe the degradation point and thermal behavior of HDPE. Fig. 2 is the TGA result generated on the HDPE at an applied heating rate of $10{ }^{\circ} \mathrm{C} \mathrm{min}{ }^{-1}$ before cooking process. As displayed in Fig. 2, weight loss curve is smooth, with one inflection point during reaction under nitrogen atmosphere. Figure 2 also shows that the decomposed point of HDPE is about $377^{\circ} \mathrm{C}$. Decomposition reactions for the heating rate of occurred at the temperature range from $630 \mathrm{~K}$ to $850 \mathrm{~K}$.

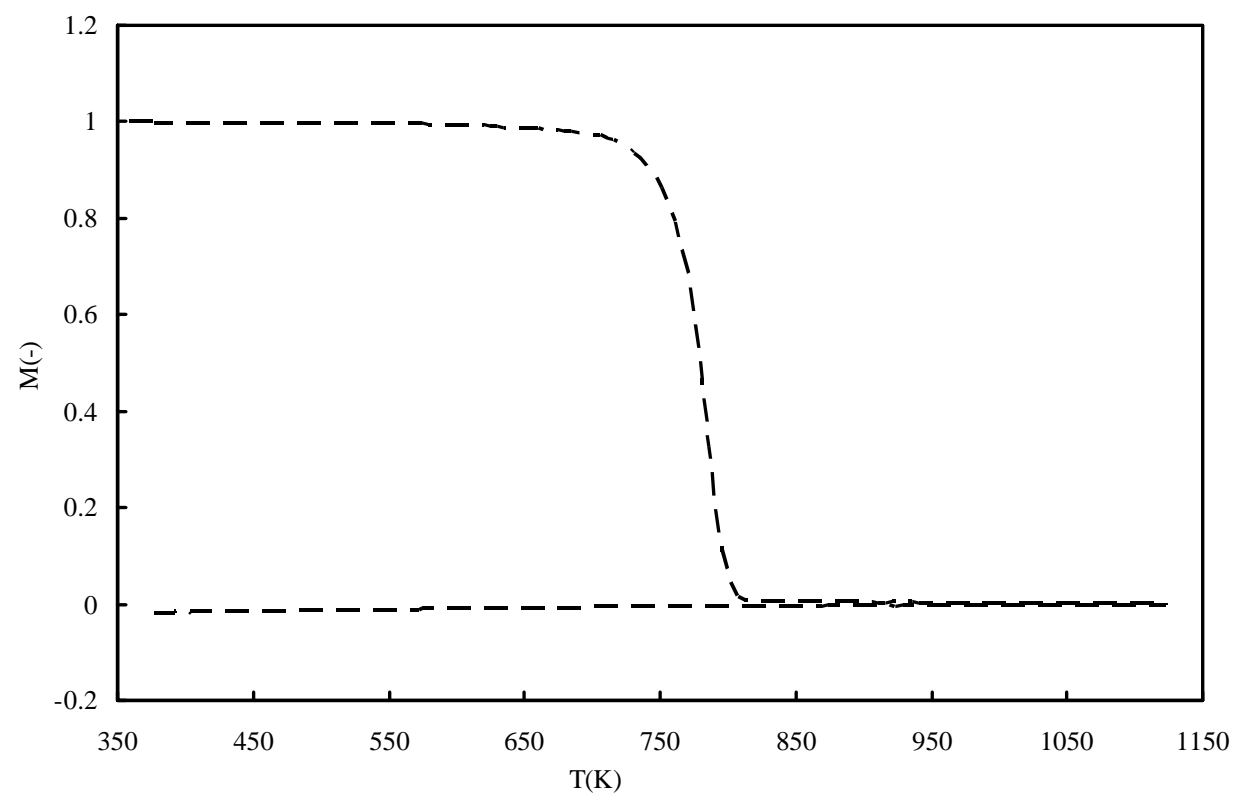

Fig. 2. The TGA curves for high density polyethylene (HDPE). 


\subsection{Dry weight changes after cooking}

We can roughly speculate the thermal characteristics of the HDPE samples by the observation of weight changes in samples. As shown in Fig. 3, there is no significant dry weight change before and after cooking process in a steaming condition of 100, 150 and $180{ }^{\circ} \mathrm{C}$. As HDPE has a melting point of $130-140{ }^{\circ} \mathrm{C}$ and a degradation point of $350-380{ }^{\circ} \mathrm{C}$ [7], the results indicate that the melting of HDPE at 150 and $180{ }^{\circ} \mathrm{C}$ has negligible effect on dry weight reduction.

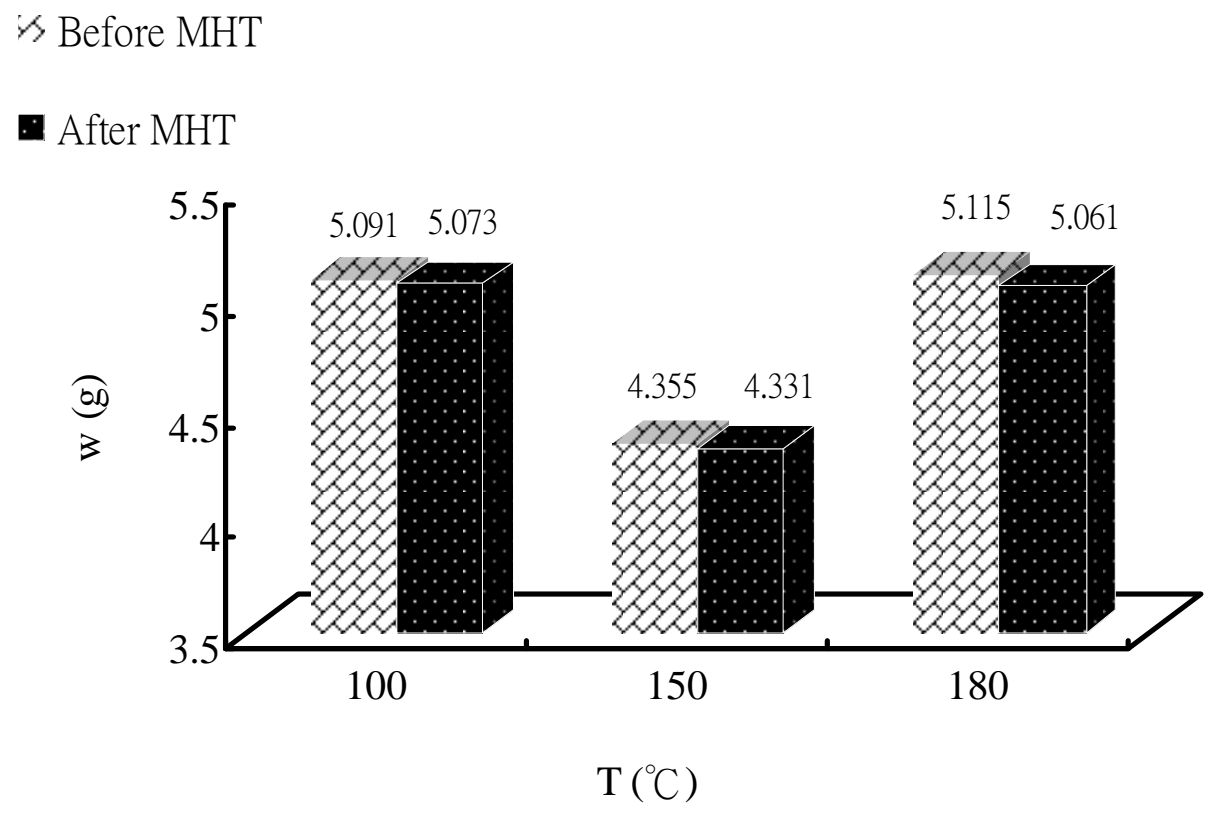

Fig. 3. Dry weight changes for HDPE before and after the MHT with saturated steam at 100, 150 and $180^{\circ} \mathrm{C}$. w: Dry weight.

\subsection{Triple component analysis}

With the analysis of triple components (including water, ash and combustible components), we can know if there's any change in the organic components of the samples. Moreover, a high quality of RDF would possess a higher value for the combustible component, and lower values for moisture and ash contents. Thus, the triple components analysis is also an important index to assess MSW if it is suitable to be utilized as RDF. The results of Fig. 4 show there is little change in the amount of combustible component existed in HDPE after cooking. The combustible component content is 99.62\% before cooking and 99.02, 98.98, $98.21 \%$ after being cooked in saturated steam condition of 100, 150 and $180{ }^{\circ} \mathrm{C}$, respectively. Therefore, we can conclude that the organic component does not reduce in those steaming conditions of 100,150 and $180{ }^{\circ} \mathrm{C}$. The results also indicate that the combustible component (>98\%) of HDPE is very high, which is larger than that of the unprocessed MSW (30-40\%). Thus, if we recycle the plastics as energy resource or RDF via MHT process, the energy utilization of MHT will be more efficient and effective than that of direct incineration treatment of MSW. 


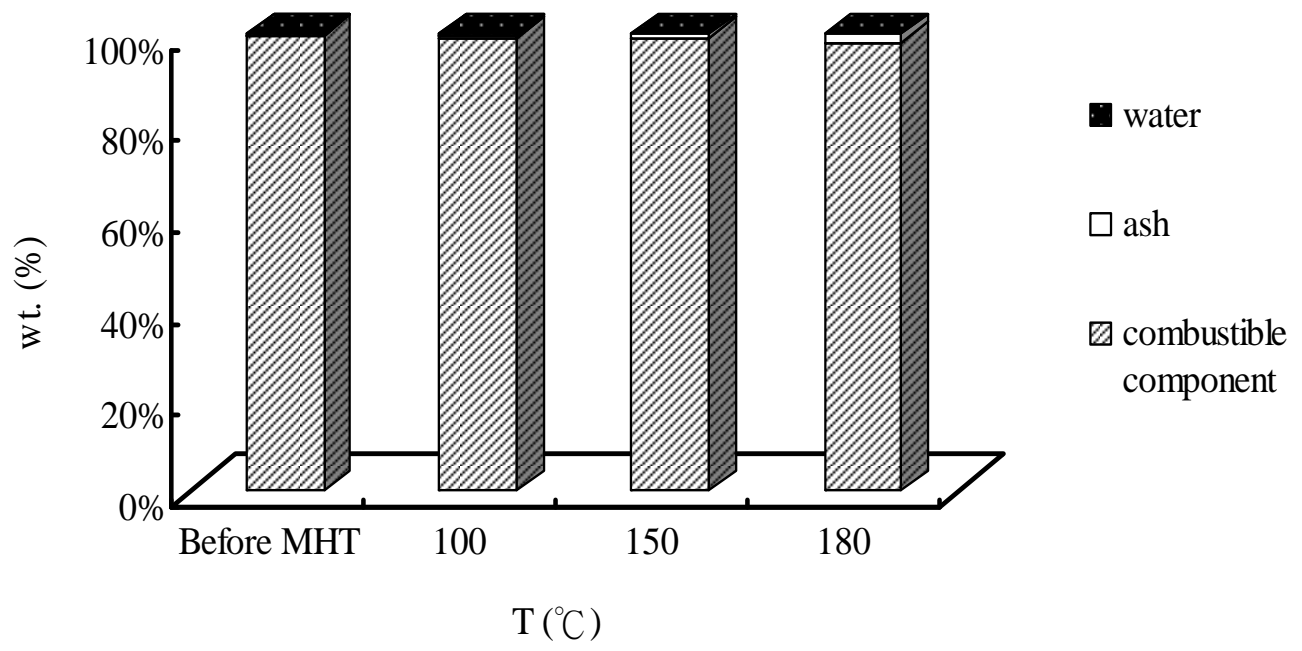

Fig. 4. Triple component analysis before and after the MHT with saturated steam at 100, 150 and 180 ${ }^{\circ} \mathrm{C}$. wt.: Weight percentage.

\subsection{True density changes}

Figure 5 indicates that with an increase of temperature of steam introduced, the true density after cooking offers increases, reaching a value of $0.983 \mathrm{~g} \mathrm{~cm}^{-3}$ at $180{ }^{\circ} \mathrm{C}$. This is due to that thermoplastic material fluidizes and swells after being heated. It then solidifies and shrinks from the melt status during cooling. Taking advantage of this characteristic, cooking process offers a very promising volume reduction effect for thermoplastics and can reduce the storage space and transportation expense drastically. Moreover, plastics soften and shrink into pellet shape with an increasing density and thus can be easily separated from other components existed in the MSW and enhancing recovery of plastics for utilization.

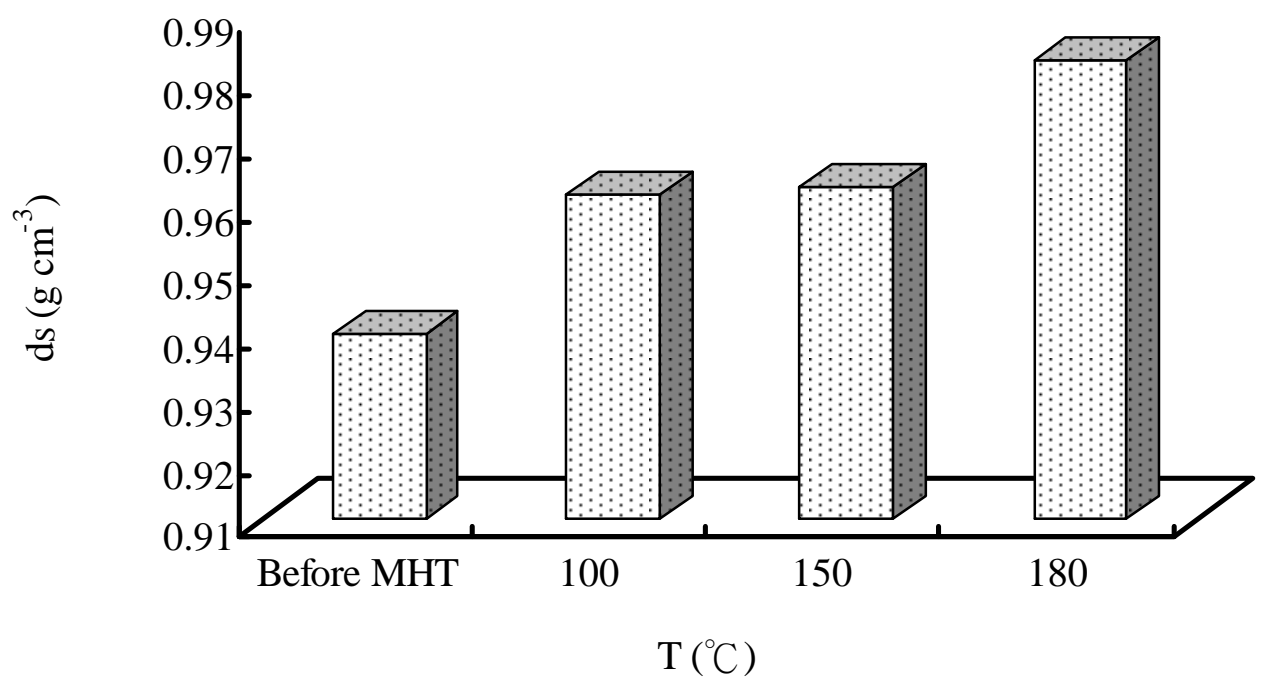

Fig. 5. True density changes before and after the MHT with saturated steam at 100, 150 and $180{ }^{\circ} \mathrm{C}$. $d_{s}$ : True density. 


\subsection{Calorific value changes}

As shown in Fig. 6, the heating values $\left(\mathrm{H}_{\mathrm{V}}\right)$ of HDPE before and after MHT are higher than that of coal of about 7000-8000 kcal kg-1 [8]. It has a value of $10654 \mathrm{kcal} \mathrm{kg}^{-1}$ before cooking and 10834,10680 and $10669 \mathrm{kcal} \mathrm{kg}^{-1}$ after cooking in saturated steam condition of 100, 150 and $180{ }^{\circ} \mathrm{C}$, respectively. We observed that $\mathrm{H}_{\mathrm{V}}$ did not have a big change for the said cooking conditions. We can therefore conclude that the cooking process does not cause the degradation or other chemical changes of HDPE which may further influence its heating value. As contrasted with the $\mathrm{H}_{\mathrm{v}}$ of $1889 \mathrm{kcal} \mathrm{kg}^{-1}$ of unprocessed MSW [4], it is clear that the separation of non-combustibles in the waste (glass and metals) can increase heating value and energy generation. From the point of view of maximization of energy recovery of MSW, MHT is a good pretreatment process for the separation of non-combustibles and energy recovery in MSW.

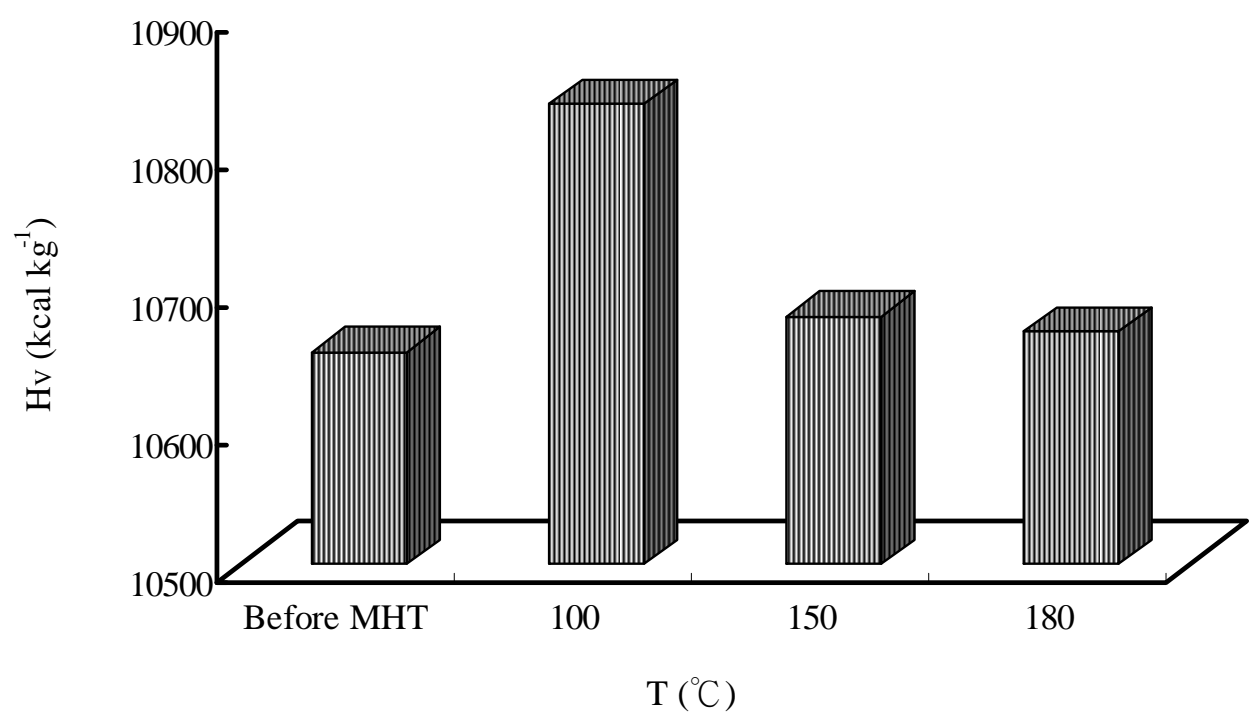

Fig. 6. Changes of heating value before and after the MHT with saturated steam at 100, 150 and 180 ${ }^{\circ} \mathrm{C}$. $H_{V}$ : Heating value.

\section{Discussion and conclusion}

In accordance with the theme of sustainable material management (SMM), the waste is viewed as the used material. The ultimate goal of SMM is targeted at total recycling and reuse without discharge of waste. To follow the appeal of SMM, this study applied the MHT for the recovery of waste plastics from waste stream, aiming at the feasibility of its recovery for the re-utilization as energy resource. As shown in Fig. 7, the recovered plastics can be directly utilized as RDF. The ferric materials and gravels can be separated via magnetic or gravity separations. The MSW containing cellulose (including organic fiber (fiber) and cotton (floc)) can partially hydrolyze after cooking to form bulk powder materials, which can help the decomposed cellulose granulation to produce renewable fuels. The treated MSW containing cellulose can be torrefied together with other agricultural and forestry materials to produce biocoal, or use as an agricultural soil improvement materials. Therefore, we can summarize that MHT has the following advantages including the disinfection, deodorization of the waste, ease of sorting and recycling and effective utilization of resources especially for energy use. 


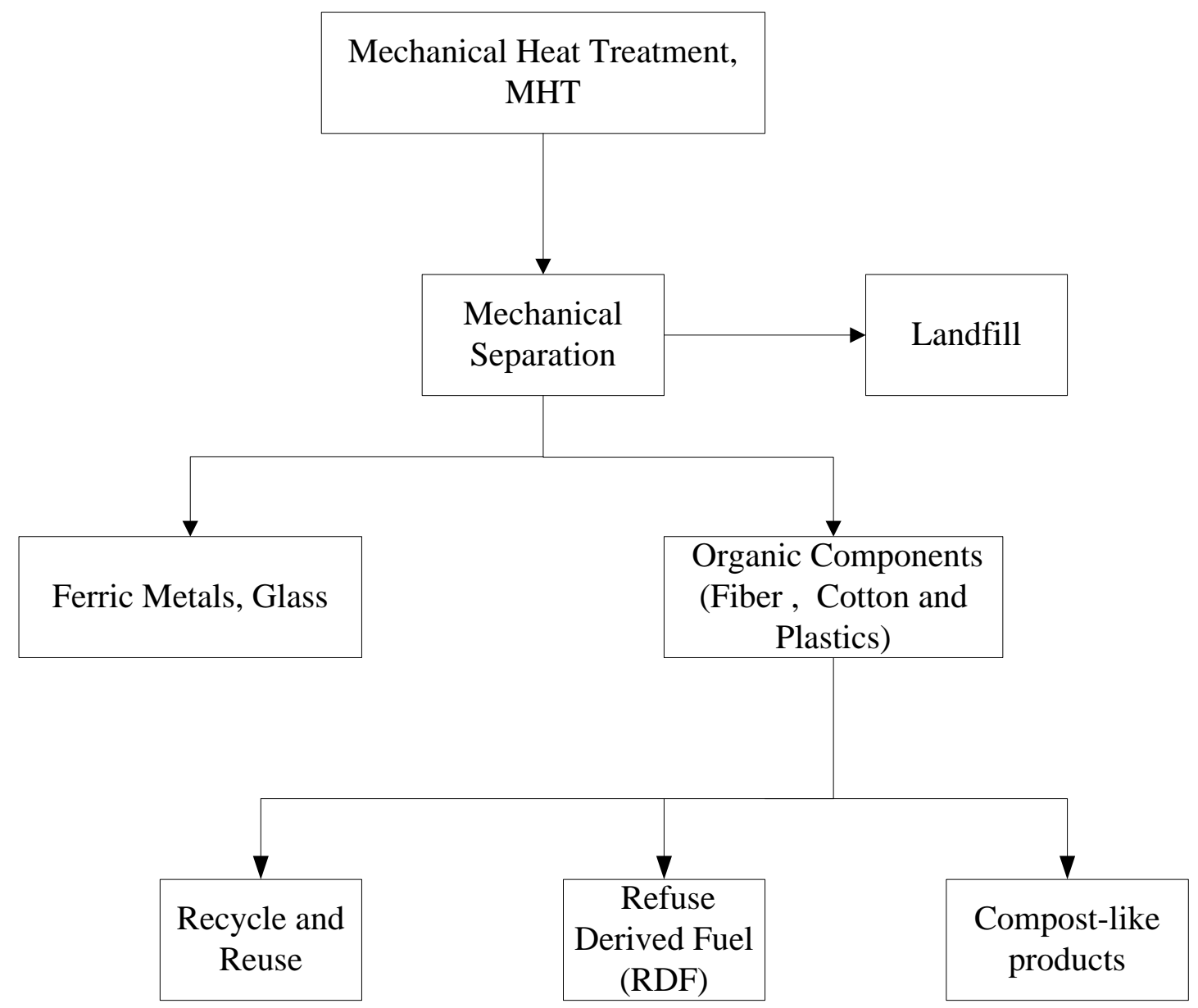

Fig. 7. The flow chart of Mechanical heat treatment (MHT) used for municipal solid waste (MSW).

Among these resources, the treated plastics can be easily separated, stored and transported, because the plastics are deformed and shrank into pellet shape with an increasing density after MHT. Further, the chemical characteristics, such as dry weight, combustible component and $\mathrm{H}_{\mathrm{V}}$, do not have significant changes for the said cooking conditions, which can be concluded that MHT is a good and interesting process to completely recycle the plastics as energy resource if compared to incineration treatment. The information obtained in this study is useful for the rational design and proper operation of MHT system for treating the used plastics in the MSW and separating it for the re-utilization as energy resource.

We can draw the following critical conclusions through the experimental results cited above.

(1) With the cooking technique, it can achieve a volume and size reduction of HDPE.

(2) The true density of HDPE reaches a high value of $0.983 \mathrm{~g} \mathrm{~cm}^{-3}$ with a $180{ }^{\circ} \mathrm{C}$ saturated steam condition.

(3) HDPE has a very high calorific value and exhibits no significant changes in dry weight, triple component composition and calorific value after MHT. It is very suitable to be used as RDF.

(4) As compared with the calorific value and combustible component of HDPE and unprocessed MSW, the energy utilization of MHT will be more efficient and effective than that of direct incineration treatment of MSW. 


\section{Acknowledgments}

The authors are grateful for the financial supports provided by the National Science Council and the Environmental Protection Administration of Taiwan.

\section{References}

[1] R. Longcake, Waste Treatment Technologies, City of Bradford Metropolitan District Council, 2007, pp. 8.

[2] Y.S. Hung, Y.H. Chen, N.C. Shang, C.H. Chang, T.L. Lu, C.Y. Chang and J.L. Shie, Comparison of biodiesels produced from waste and virgin vegetable oils, Sustainable Environment Research 20, 2010, pp. 417-422.

[3] J.L. Shie, C.Y. Chang, C.C. Tzeng, P.Y. Hung, C.C. Chang, S.W. Chiang, J.Y. Tseng, W.K. Tu, M.H. Yuan, H.Y. Li and Y.J. Yu, Water-enhanced reforming of syngas to fuels and chemicals in a batch reactor, Journal of Environmental Engineering and Management 20, 2010, pp. 181-187.

[4] Taiwan Environmental Protection Administration (TEPA), Database of Taiwan's Environmental Statistics, http://210.69.101.110/WEBSTATIS/webindex.htm (accessed in Sep. 2009). (In Chinese)

[5] A. Papageorgiou, J.R. Barton, A. Karagiannidis, Assessment of the greenhouse effect impact of technologies used for energy recovery from municipal waste: a case for england, Journal of Environmental Management 90, 2009, pp. 2999-3012.

[6] A. Garg, R. Smith, D. Hill, N. Simms and S. Pollard, Wastes as co-fuels: the policy framework for solid recovered fuel (SRF) in Europe, with UK implications, Environmental Science \& Technology 41, 2007, pp. 4868-4874.

[7] Q.B. Wang, H.J. Huang, B.H. Xie, W. Yang, M.B. Yang, Thermal degradation of HDPE in a batch pressure reactor: reaction time and mechanical stirring effect, Journal of Macromolecular Science, Part A 47, 2010, pp. 1123-1129.

[8] Wikipedia, Coal, http://en.wikipedia.org/wiki/Coal. 